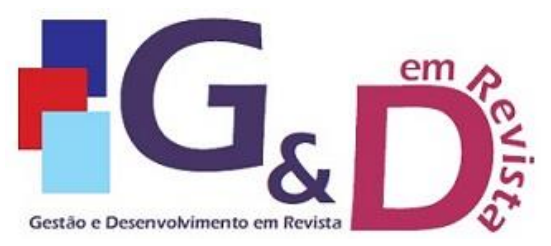

Gestão e Desenvolvimento em Revista

V. 7, N. 1, jan-jun/2021, p. 03-14.

ISSN online: $2446-8738$

Artigo recebido em: 18/02/2021

Artigo aprovado em: 19/04/2021

\title{
COMPRAS PÚBLICAS COMO INSTRUMENTO DE DESENVOLVIMENTO LOCAL
}

\author{
Roger Alexandre Rossoni \\ Graduação em Ciências Econômicas. Mestrado em Administração. Atualmente é \\ E-mail: roger.rossoni@gmail.com
}

\section{Resumo}

Segundo estimativa do Ministério do Planejamento, Orçamento e Gestão, as compras públicas movimentam valores de aproximadamente $15 \%$ do PIB. O presente estudo pretende verificar empiricamente o impacto das compras públicas como instrumento de desenvolvimento socioeconômico nos municípios do Paraná. Por meio da utilização da regressão linear múltipla aplicada a dados referente às compras públicas dos municípios paranaenses em 2013, variáveis socioeconômicas e ao Índice IPARDES de Desempenho Municipal (IPDM), foi verificado o efeito das compras públicas no processo de desenvolvimento no Estado do Paraná. O modelo estimado demonstra que as compras públicas, quando realizadas em fornecedores locais, implicaram em um maior IPDM nos municípios paranaenses. Apesar do modelo demonstrar uma relação positiva entre a realização de compras públicas em fornecedores locais e o IPDM, verifica-se que o impacto das compras públicas no IPDM é de pequena proporção. Desta forma, a utilização das compras públicas como fator estratégico para desenvolvimento socioeconômico apresenta suas limitações. A utilização das compras públicas como indutor do desenvolvimento local necessita de um maior debate e da adequação da legislação vigente referente ao processo de licitação.

Palavras-chave: Desenvolvimento. Compras Públicas. Paraná.

\begin{abstract}
According to estimates by the Ministry of Planning, Budget and Management, public procurement accounts for approximately $15 \%$ of GDP. This study aims to empirically verify the impact of public procurement as socioeconomic development tool in Paraná municipalities. Through the use of multiple linear regression applied to data regarding the procurement of municipalities in 2013, socioeconomic variables and the Municipal Performance IPARDES Index (IPDM), it was verified the effect of public procurement in the development process in the State of Paraná. The estimated model shows that public procurement, when carried out at local suppliers, implied in a higher IPDM in the municipalities of Paraná. Although the model demonstrates a positive relationship between public procurement in local suppliers and IPDM, it can be seen that the impact of public procurement on IPDM is small. Thus, the use of public procurement as a strategic factor for socioeconomic development have limitations. The use of public procurement as an inducer of local development requires a greater debate and the adequacy of the legislation in force regarding about the bidding process.
\end{abstract}

Keywords: Development. Public Procurement. Paraná. 


\section{INTRODUÇÃO}

O processo de compras realizadas por entidades públicas brasileiras é regido conforme a Lei ำ $8.666 / 93$, que estabelece normas sobre o processo de contratação de bens, serviços, alienações e locações para os órgãos de administração pública, como a União, Estados, Distrito Federal e Municípios, e pelo capítulo 1 da Lei no 13.303/16, que discorre sobre o processo de compras realizados por empresas públicas e de sociedades de economia mista.

Segundo estimativa do Ministério do Planejamento, Orçamento e Gestão (BRASIL, 2012), as compras públicas movimentam valores de aproximadamente $15 \%$ do PIB. Considerando o grande volume de recursos aplicados, o setor público pode ser considerado como uma oportunidade de mercado para diversos ramos do setor privado.

Além do fomento a atividade privada, as compras públicas vêm sendo utilizadas como fator estratégico na execução de políticas. Pode-se citar o fomento a inovação, consumo de produtos ambientalmente sustentáveis e fortalecimento do pequeno produtor agrícola como exemplos.

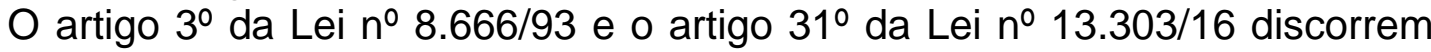
que o processo de compra pública visa garantir a proposta mais vantajosa para a administração e a promoção do desenvolvimento nacional sustentável. Destaca-se que não há uma definição objetiva do termo "proposta mais vantajosa", sendo papel dos responsáveis pelo processo licitatório realizarem um julgamento objetivo e justificado quanto à proposta mais vantajosa. No tocante ao desenvolvimento nacional sustentável, o artigo $4^{\circ}$ do Decreto 7.746/12 discorre sobre critérios e práticas para promoção do desenvolvimento nacional sustentável. Nota-se a existência de critérios relacionados à sustentabilidade ambiental, como a preferência por materiais de maior eficiência energética e de baixo impacto sobre os recursos ambientais, bem como critérios relacionados ao desenvolvimento econômico local, como a preferência por materiais de origem local e a maior geração de empregos, preferencialmente com mão de obra local.

Portanto, nota-se, na promoção do desenvolvimento legal sustentável, um possível instrumento de desenvolvimento econômico via compras públicas locais. A eficiência das compras públicas impacta no bem-estar de toda a sociedade. A contratação de fornecedores locais, observando o critério de proposta mais vantajosa, mantém os recursos financeiros na economia local, multiplicando seu efeito em forma de emprego e renda.

O presente estudo pretende verificar empiricamente o impacto das compras públicas como instrumento de desenvolvimento socioeconômico nos municípios do Paraná. Por meio da utilização da regressão linear múltipla aplicada a dados referente às compras públicas dos municípios paranaenses em 2013 e ao Índice IPARDES de Desempenho Municipal (IPDM), foi verificado o efeito das compras públicas no processo de desenvolvimento no Estado do Paraná.

O presente estudo está divido em cinco seções: Introdução; Revisão de literatura - que aborda estudos referente compras públicas e dados do Paraná; Metodologia - aborda o método aplicado e as variáveis utilizadas; Resultados apresenta os resultados da pesquisa; e Conclusão - apresenta as considerações finais da pesquisa e indicação para estudos futuros. 


\section{REVISÃO DE LITERATURA}

Conforme exposto na introdução, as compras públicas podem ser utilizadas como instrumento estratégico na execução de políticas. Esta seção apresenta alguns trabalhos desenvolvidos referente ao papel das compras públicas como fator de indução.

Em 2004, a European Comission (2005) apresentou um documento abordando o fomento a inovações por meio das compras públicas. Edler et al. (2012) destacam que diversos países europeus recentemente adotaram uma política de fomento a inovações baseada na demanda do setor público. As inovações são fomentadas pelo setor público por meio de incentivos com o objetivo de suprir necessidades sociais e que estimulem o desenvolvimento do mercado de determinadas regiões.

Lopes e Oliveira (2012) abordam a questão ambiental nas compras públicas. Segundo os autores, a Lei 12.349 apresentou diversos avanços no processo de compras públicas ao contexto socioambiental. Destaca-se o caso do governo da Bahia que incorporou as compras públicas de forma estratégica, gerando ganhos econômicos e sociais. O autor aponta a necessidade de o Estado intervir na disseminação das compras sustentáveis para que ocorra uma mudança nos padrões de consumo.

Caldas e Nonato (2013), por meio de estudo de caso, analisam o caso do município do Apucarana, percursor na utilização das compras públicas municipais no fortalecimento dos agricultores familiares por meio da compra de alimentos para a merenda escolar. Os resultados indicam que os municípios podem ser capazes de promover políticas públicas por meio das compras públicas. Destaca-se o potencial de ampliar as estratégias de compras para demais gêneros.

Chaves, Bertassi e Silva (2019) analisaram a influência das micro e pequenas empresas locais e regionais no processo licitatório de uma universidade pública mineira, entre 2015 e 2017. Utilizando de estatística descritiva, com dados secundários, verificou-se que, apesar do aumento no número de micro e pequenas empresas contratadas, as compras de micro e pequenas empresas representam menos de $1 \%$ das compras realizadas. Os autores indicam a necessidade de avanços normativos e da intensificação das pesquisas acerca da utilização da compra pública como política de desenvolvimento.

Os estudos citados acima demostram o potencial das compras públicas como indutor de políticas públicas. Apesar da ampla discussão acerca da utilização das compras públicas para diversas finalidades, inclusive desenvolvimento, carecem de estudos quantitativos para complementar a discussão. A seção seguinte apresenta dados referente compras públicas no Estado do Paraná.

\subsection{Compras públicas no Paraná}

Segundo levantamento do SEBRAE (2016), os municípios paranaenses gastaram $R \$ 8,75$ bilhões em compras públicas no ano de 2013. Deste valor, $R \$ 4,32$ bilhões foram gastos em fornecedores locais, representando $49,4 \%$ do valor gasto em compras públicas. O gasto per capto médio do Estado do Paraná em 2013 foi de $\mathrm{R} \$ 795,46$. Contudo, ressalta-se que o Paraná apresenta 399 municípios e o gasto em compras públicas varia de acordo com o município. 
A Tabela 1 apresenta os 10 municípios com maior gasto total e local por habitante. O município de Itaipulândia apresentou maior gasto total por habitante em 2013 , com um gasto de $\mathrm{R} \$ 2.848,80$ por habitante, valor $258 \%$ maior que a média estadual. Da mesma forma, o município de Itaipulândia apresentou o maior gasto local por habitante, com gasto de $\mathrm{R} \$ 1.291,60$ realizado em fornecedores locais.

Tabela 1 - Municípios paranaenses com maiores gastos em compras públicas por habitante em 2013.

\begin{tabular}{|c|c|c|c|c|c|c|}
\hline Município & Habitantes & $\begin{array}{l}\text { Gasto Total por } \\
\text { Habitante }\end{array}$ & Município & Habitantes & \multicolumn{2}{|c|}{$\begin{array}{l}\text { Gasto Local } \\
\text { por Habitante }\end{array}$} \\
\hline Itaipulândia & 9.869 & $\mathrm{R} \$ 2.848,80$ & Itaipulândia & 9.869 & $\mathrm{R} \$$ & $.291,60$ \\
\hline $\begin{array}{l}\text { Entre Rios do } \\
\text { Oeste }\end{array}$ & 4.202 & $\mathrm{R} \$ 2.805,89$ & $\begin{array}{c}\text { Entre Rios do } \\
\text { Oeste }\end{array}$ & 4.202 & $\mathrm{R} \$$ & $.023,78$ \\
\hline $\begin{array}{l}\text { Saudade do } \\
\text { Iguaçu }\end{array}$ & 5.293 & $\mathrm{R} \$ 2.506,30$ & Pato Branco & 77.230 & $\mathrm{R} \$$ & 964,10 \\
\hline Jardim Olinda & 1.424 & $R \$ 2.446,36$ & $\begin{array}{c}\text { Bom Sucesso } \\
\text { do Sul }\end{array}$ & 3.372 & $\mathrm{R} \$$ & 880,55 \\
\hline $\begin{array}{c}\text { Bom Sucesso } \\
\text { do Sul }\end{array}$ & 3.372 & $\mathrm{R} \$ 2.442,40$ & $\begin{array}{l}\text { Saudade do } \\
\text { Iguaçu }\end{array}$ & 5.293 & $\mathrm{R} \$$ & 846,15 \\
\hline $\begin{array}{c}\text { Pinhal de São } \\
\text { Bento }\end{array}$ & 2.724 & $\mathrm{R} \$ 2.279,89$ & Pato Bragado & 5.170 & $\mathrm{R} \$$ & 813,32 \\
\hline Sulina & 3.366 & $\mathrm{R} \$ 2.157,79$ & Miraselva & 1.896 & $\mathrm{R} \$$ & 773,74 \\
\hline Pato Bragado & 5.170 & $\mathrm{R} \$ 2.114,32$ & Curitiba & 1.848 .946 & $\mathrm{R} \$$ & 726,64 \\
\hline $\begin{array}{c}\text { Boa Esperança } \\
\text { do Iguaçu }\end{array}$ & 2.763 & $\mathrm{R} \$ 2.043,24$ & Mangueirinha & 17.402 & $\mathrm{R} \$$ & 721,63 \\
\hline $\begin{array}{c}\text { Serranópolis do } \\
\text { Iguaçu }\end{array}$ & 4.667 & $\mathrm{R} \$ 2.013,49$ & Mercedes & 5.316 & $\mathrm{R} \$$ & 708,98 \\
\hline
\end{tabular}

Fonte: Elaborado pelo autor com dados do SEBRAE (2016).

Verifica-se que entre os dez municípios com maior gasto em compras públicas por habitante, todos são municípios de pequeno porte, com menos de 10.000 habitantes. Ao analisar os municípios com maior gasto em fornecedores locais, observa-se que três municípios com mais de 10.000 habitantes se apresentam entre os dez municípios com maior gasto.

Os municípios de pequeno porte apresentam um mercado mais limitado, possivelmente sendo este um dos fatores que explicam o menor gasto em fornecedores locais apesar do maior gasto total. Desta forma, é necessário analisar o percentual dos gastos realizados em fornecedores locais para verificar quais municípios apresentam maior participação de fornecedores locais.

A Tabela 2 apresenta os 10 municípios com maior porcentagem dos gastos em compras públicas realizados em fornecedores locais. De forma distinta do gasto total por habitante, os municípios com maior percentual gasto em fornecedores locais são de diversos portes. O município de Guarapuava realizou $79,19 \%$ dos 
gastos em compras públicas em fornecedores locais, sendo o município com maior percentual de gastos em fornecedores locais.

Tabela 2 - Municípios paranaenses com maior porcentagem de compras públicas em fornecedores locais em 2013.

\begin{tabular}{ccc}
\hline Município & Habitantes & \% de gastos locais \\
\hline Guarapuava & 175.779 & $79,13 \%$ \\
\hline Campo Mourão & 91.648 & $75,11 \%$ \\
\hline Palmital & 14.780 & $73,36 \%$ \\
\hline Pato Branco & 77.230 & $72,18 \%$ \\
\hline Londrina & 537.566 & $71,91 \%$ \\
\hline Umuarama & 106.387 & $71,91 \%$ \\
\hline Quedas do Iguaçu & 32.393 & $71,17 \%$ \\
\hline Curitiba & 1.848 .946 & $70,81 \%$ \\
\hline Cianorte & 75.360 & $70,10 \%$ \\
\hline Maringá & 385.753 & $65,75 \%$ \\
\hline
\end{tabular}

Fonte: Elaborado pelo autor com dados do SEBRAE (2016).

Verifica-se que entre os dez municípios com maior porcentagem, quatro estão entre os dez municípios de maior população do Paraná. Desta forma, os dados indicam que possivelmente municípios de maior porte, por possuírem maior mercado, apresentam maior participação de fornecedores locais nas compras públicas. Contudo, esta afirmação não pode ser generalizada. Palmital e Quedas do Iguaçu, apesar da menor população, apresentaram alta participação de fornecedores locais nas compras públicas.

Os municípios com maior gasto por habitante são de pequeno porte, indicando uma possível relação com o menor número de habitantes. Por outro lado, o gasto em fornecedores locais decorre da participação dos fornecedores locais nas compras públicas. Desta forma, sendo maior a disponibilidade de bens e serviços ofertados, maior é a possibilidade de ofertar ao setor público.

\section{METODOLOGIA}

Este estudo apresenta uma abordagem quantitativa, com a utilização da regressão linear. Segundo Fávero et al. (2009), a regressão linear visa identificar a relação entre duas ou mais variáveis explicativas sobre uma varável dependente.

A aplicação correta da regressão implica no atendimento dos seguintes pressupostos: normalidade de resíduos; ausência de auto correlação serial dos resíduos; homocedasticidade dos resíduos; e linearidade dos coeficientes. Atendendo todos os pressupostos, a regressão é considerada válida.

\subsection{Variáveis selecionadas}

Como indicador de desenvolvimento foi escolhido o IPDM, calculado pelo 
Instituto Paranaense de Desenvolvimento Econômico e Social (IPARDES). O IPDM é dividido em três dimensões: Renda e Emprego, Educação e Saúde. Desta forma, o IPDM pode ser utilizado como indicador do nível de desenvolvimento do município.

O IPDM apresenta valores entre 0 e 1 . A variável IPDM foi transformada para uma escala de 0 a 100 , para evitar que o resultado do coeficiente de regressão seja 0 , devido a diferença de escala entre as variáveis.

Como indicadores de compras públicas, foi selecionado o valor total gasto em compras públicas, o valor gasto em compras públicas de fornecedores locais e o percentual dos gastos em compras públicas realizado em fornecedores locais. O gasto total e o gasto em fornecedores locais foram analisados de forma per capita. $O$ número de habitantes foi coletado do Instituto Brasileiro de Geografia (IBGE, 2016).

Foram incluídas no modelo variáveis socioeconômicas com o intuito de aumentar o poder de predição do modelo. Desta forma, espera-se obter maior precisão em mensurar a influência das compras públicas no processo de desenvolvimento socioeconômico.

$\mathrm{O}$ indicador de desenvolvimento e as variáveis relacionadas às compras públicas são referentes ao ano de 2013. As variáveis socioeconômicas utilizadas se referem ao ano de 2010. Ressalta-se que a defasagem temporal dos dados decorre da inexistência de dados para o período de 2013 e, que por se tratarem de indicadores populacionais, não se sofrem significativas alterações em curto período de tempo. Desta forma, os dados de 2010 podem ser utilizados para representar as características populacionais dos municípios paranaenses.

O Quadro 1 apresenta as variáveis aplicadas na regressão. Os dados referentes às compras públicas foram coletados do programa COMPRA-PR, realizado pelo Serviço Brasileiro de Apoio à Micro e Pequena Empresa (SEBRAE, 2016). O IPDM e os indicadores socioeconômicos foram coletados da base de dados do IPARDES (2016).

\subsubsection{Procedimentos}

O tratamento estatístico foi realizado com auxílio do software SPSS $\AA$ versão 18.0. Em primeiro momento, foram coletados os dados e calculados o gasto total e o gasto total local por habitante. Em seguida, foi aplicada a regressão linear múltipla com utilização do método stepwise. As variáveis que apresentaram significância menor de 0,05 foram incluídas no modelo de regressão. A qualidade de explicação da realidade do modelo foi avaliada pelo coeficiente de determinação ajustado (R_a^2).

Após verificado quais as variáveis se mostraram significantes em relação à variável dependente, foram realizados testes para verificar a presença de multicolinearidade, a ausência de autocorrelação serial nos resíduos, a linearidade dos coeficientes, a homocesdaticidade dos resíduos e a normalidade dos resíduos. 


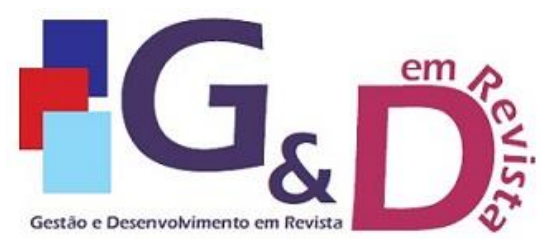

Gestão e Desenvolvimento em Revista V. 7, N. 1, jan-jun/2021, p. 03-14.

ISSN online: $2446-8738$

Artigo recebido em: 18/02/2021

Artigo aprovado em: 19/04/2021

Quadro 1 - Descrição das Variáveis da Pesquisa

\begin{tabular}{|c|c|c|}
\hline Atividades & Variável & Descrição \\
\hline \multirow{3}{*}{$\begin{array}{l}\text { Compras } \\
\text { Públicas } \\
\text { (Variáveis } \\
\text { explicativas) }\end{array}$} & Gasto local por habitante & Gasto em compras públicas por habitante \\
\hline & Gasto total por habitante & $\begin{array}{l}\text { Gasto em compras públicas em fornecedores } \\
\text { locais por habitante }\end{array}$ \\
\hline & Percentual de gastos locais & $\begin{array}{l}\text { Percentual dos gastos públicos realizados em } \\
\text { fornecedores locais }\end{array}$ \\
\hline \multirow{9}{*}{$\begin{array}{l}\text { Indicadores } \\
\text { Socioeconômicos } \\
\text { (Variáveis } \\
\text { explicativas) }\end{array}$} & Grau de Urbanização (\%) & $\begin{array}{l}\text { Percentagem da população da área urbana } \\
\text { em relação à população total. }\end{array}$ \\
\hline & Índice de Idosos (\%) & $\begin{array}{l}\text { Mede a relação entre o número de pessoas } \\
\text { idosas ( } 65 \text { anos e mais) e o número de } \\
\text { pessoas nos grupos etários mais jovens } \\
\text { (menores de } 15 \text { anos de idade). }\end{array}$ \\
\hline & $\begin{array}{l}\text { Probabilidade de Sobrevivência } \\
\text { até } 60 \text { anos (\%) }\end{array}$ & $\begin{array}{l}\text { Probabilidade de uma criança recém-nascida } \\
\text { viver até os } 60 \text { anos }\end{array}$ \\
\hline & $\begin{array}{l}\text { Renda Média Domiciliar per } \\
\text { Capita }(R \$ 1,00)\end{array}$ & $\begin{array}{l}\text { Média das rendas domiciliares per capita das } \\
\text { pessoas residentes }\end{array}$ \\
\hline & $\begin{array}{l}\text { Taxa de Analfabetismo de } 15 \\
\text { anos ou mais (\%) }\end{array}$ & Percentual de pessoas analfabetas \\
\hline & Taxa de Pobreza (\%) & $\begin{array}{l}\text { Proporção dos indivíduos com renda } \\
\text { domiciliar per capita igual ou inferior a } R \$ \\
140,00 \text { mensais, em reais de agosto de } 2010\end{array}$ \\
\hline & $\begin{array}{l}\text { Proporção de Extremamente } \\
\text { Pobres (\%) }\end{array}$ & $\begin{array}{l}\text { Proporção dos indivíduos com renda } \\
\text { domiciliar per capita igual ou inferior a } R \$ \\
70,00 \text { (setenta reais) mensais, em reais de } \\
\text { agosto de } 2010\end{array}$ \\
\hline & $\begin{array}{l}\text { Taxa de Aprovação no Ensino } \\
\text { Fundamental (\%) }\end{array}$ & $\begin{array}{l}\text { Proporção de alunos da matrícula total, em } \\
\text { cada série / ano do ensino fundamental, no } \\
\text { ano, que são aprovados. }\end{array}$ \\
\hline & $\begin{array}{l}\text { Taxa de Aprovação no Ensino } \\
\text { Médio (\%) }\end{array}$ & $\begin{array}{l}\text { Proporção de alunos da matrícula total, em } \\
\text { cada série / ano do ensino médio, no ano, } \\
\text { que são aprovados. }\end{array}$ \\
\hline $\begin{array}{l}\text { Desenvolvimento } \\
\text { Municipal } \\
\text { (Variável } \\
\text { dependente) }\end{array}$ & IPDM & Índice IPARDES de Desempenho Municipal \\
\hline
\end{tabular}

Fonte: Elaborado pelos autores

\section{APRESENTAÇÃO E ANÁLISE dOS RESULTADOS}

As variáveis explicativas foram selecionadas de acordo com o seu nível de significância em relação a variável dependente. O percentual de gastos locais, o gasto total por habitante, o grau de urbanização, o índice de idosos, possibilidade de sobrevivência até sessenta anos e a taxa de aprovação no ensino médio não foram consideradas significantes pois apresentaram significância maior que 0,050, conforme Tabela 3. Variáveis não significantes foram excluídas do modelo. Desta 
forma, o modelo considerou cinco variáveis significantes, sendo elas: o gasto local por habitante; taxa de pobreza; taxa de aprovação no ensino fundamental; gasto local por habitante; e a taxa de analfabetismo.

Tabela 3 - Significância das Variáveis Independentes

\begin{tabular}{ll}
\hline \multicolumn{1}{c}{ Variáveis independentes } & Significância \\
\hline Renda Média Domiciliar per capita (R\$ 1,00) & 0,000 \\
\hline Taxa de Pobreza (\%) & 0,000 \\
\hline Taxa de Aprovação no Ensino Fundamental (\%) & 0,000 \\
\hline Gasto local por habitante & 0,000 \\
\hline Taxa de Analfabetismo de 15 anos ou mais (\%) & 0,000 \\
\hline Grau de Urbanização (\%) & 0,438 \\
\hline Índice de Idosos (\%) & 0,619 \\
\hline Probabilidade de Sobrevivência até 60 anos (\%) & 0,695 \\
\hline Taxa de Aprovação no Ensino Médio (\%) & 0,720 \\
\hline Gasto total por habitante & 0,518 \\
\hline Percentual de gastos locais & 0,625
\end{tabular}

Fonte: Elaborada pelos autores com base nos resultados da pesquisa.

Excluídas as variáveis não significantes, o modelo de regressão realizado apresentou um coeficiente de explicação ajustado ( $\left.R \_a^{\wedge} 2\right)$ de 0,616, conforme Tabela 4. Segundo este indicador, $61,6 \%$ das variações do IPDM são explicadas pelas variáveis utilizadas.

Tabela 4 - Sumário do Modelo
$\mathbf{R}$
$\boldsymbol{R}^{2}$
$R_{a}^{2}$
Erro Padrão da Estimativa

$0,788 \quad 0,620 \quad 0,616 \quad 3,81342$

Fonte: Elaborada pelos autores com base nos resultados da pesquisa.

A Tabela 5 apresenta os resultados do Teste ANOVA que analisa a significância da regressão. $O$ modelo de regressão proposto é significativo, pois apresentou o sig. (nível de significância) de 0,000, inferior a 0,050. Desta forma, 0 modelo com um todo é considerado estatisticamente significativo. 


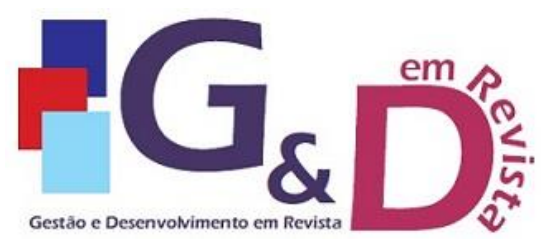

Gestão e Desenvolvimento em Revista V. 7, N. 1, jan-jun/2021, p. 03-14.

ISSN online: $2446-8738$

Artigo recebido em: 18/02/2021

Artigo aprovado em: 19/04/2021

Tabela 5 - Teste ANOVA da Regressão

\begin{tabular}{|c|c|c|c|c|c|}
\hline Modelo & $\begin{array}{c}\text { Soma dos } \\
\text { Quadrados }\end{array}$ & G.L & $\begin{array}{c}\text { Média dos } \\
\text { Quadrados }\end{array}$ & F & Sig. \\
\hline Regressão & 9341,978 & 5 & 1868,396 & 128,481 & 0,000 \\
\hline Residual & 5715,064 & 393 & 14,542 & & \\
\hline Total & 15057,043 & 398 & & & \\
\hline
\end{tabular}

Fonte: Elaborada pelos autores com base nos resultados da pesquisa.

Conforme Tabela 6, na qual consta o resumo dos resultados dos testes dos pressupostos da regressão, têm-se as seguintes conclusões:

- Multicolinearidade - o pressuposto foi atendido. O Variance Inflation Factor (VIF) apresentou valores abaixo de 10 para todos as variáveis, representando um nível de multicolinearidade aceitável.

- Ausência de autocorrelação serial - o pressuposto foi atendido. O resultado do teste de Durbin-Watson (DW) foi de 1,982, o qual está situado na parte central da tabela da estatística DW, na região de III, área de ausência de autocorrelação;

- Normalidade - o pressuposto foi atendido. O resultado do teste Kolmogorov - Smirnov foi de 0,605 , aceitando a hipótese nula de que a distribuição da série testada é normal;

- Homocedasticidade - o pressuposto foi atendido. O resultado do teste Pesarán-Pesarán foi de 0,285 , aceitando a hipótese nula que os resíduos são homoscedásticos.

Tabela 6 - Pressupostos da regressão

\begin{tabular}{|c|c|c|c|}
\hline \multirow{2}{*}{ Pressuposto } & & \multicolumn{2}{|c|}{ Testes/Resultados } \\
\hline & & Tolerância & VIF \\
\hline \multirow{5}{*}{ Multicolinearidade } & Renda Média Domiciliar per Capita $(\mathrm{R} \$ 1,00)$ & ,352 & 2,841 \\
\hline & Taxa de Pobreza (\%) &, 582 & 1,719 \\
\hline & Taxa de Aprovação no Ensino Fundamental (\%) & ,523 & 1,913 \\
\hline & Gasto local por habitante & ,957 & 1,045 \\
\hline & Taxa de Analfabetismo de 15 anos ou mais (\%) & ,863 & 1,159 \\
\hline \multicolumn{2}{|c|}{ Ausência de autocorrelação serial } & $\begin{array}{l}\text { Durbin- } \\
\text { Watson }\end{array}$ & 1,982 \\
\hline \multicolumn{2}{|l|}{ Normalidade } & $\begin{array}{l}\text { Kolmogorov- } \\
\text { Smirnov }\end{array}$ & 0,605 \\
\hline \multicolumn{2}{|c|}{ Homoscedasticidade } & $\begin{array}{l}\text { Pesarán- } \\
\text { Pesarán }\end{array}$ & 0,285 \\
\hline
\end{tabular}

Fonte: Elaborada pelos autores com base nos resultados da pesquisa.

A Tabela 7 apresenta o coeficiente de cada variável do modelo. A renda média, a taxa de aprovação no ensino fundamental e o gasto local por habitante 
apresentaram relação positiva com o IPDM. A taxa de pobreza e a taxa de analfabetismo de 15 anos ou mais apresentaram relação negativa com o IPDM.

Tabela 7 - Coeficientes da Regressão Final

\begin{tabular}{|c|c|c|c|c|c|}
\hline \multirow{3}{*}{ Modelo } & \multirow{2}{*}{\multicolumn{2}{|c|}{$\begin{array}{c}\text { Coeficientes não } \\
\text { Padronizados }\end{array}$}} & \multirow{3}{*}{$\begin{array}{c}\begin{array}{c}\text { Coeficientes } \\
\text { Padronizados }\end{array} \\
\text { Beta }\end{array}$} & \multirow{3}{*}{$-\mathbf{t}$} & \multirow{3}{*}{ Sig. } \\
\hline & & & & & \\
\hline & B & $\begin{array}{c}\text { Erro } \\
\text { padrão }\end{array}$ & & & \\
\hline \multirow{2}{*}{ (Constante) } & 41,923 & 4,763 & & 8,80 & ,000 \\
\hline & & & & 3 & \\
\hline \multirow{2}{*}{ Renda Média Domiciliar per Capita $(\mathrm{R} \$ 1,00)$} & 012 & ,002 & ,282 & 5,37 &, 000 \\
\hline & & & & 5 & \\
\hline \multirow{3}{*}{ Taxa de Pobreza (\%) } &,- 319 & ,037 &,- 374 & - & ,000 \\
\hline & & & & 8,70 & \\
\hline & & & & 5 & \\
\hline \multirow{2}{*}{ Taxa de Aprovação no Ensino Fundamental (\%) } & ,286 & ,048 & ,191 & 6,01 & ,000 \\
\hline & & & & 2 & \\
\hline \multirow{2}{*}{ Gasto local por habitante } & 006 & ,001 & ,156 & 4,65 & ,000 \\
\hline & & & & 1 & \\
\hline \multirow{3}{*}{ Taxa de Analfabetismo de 15 anos ou mais (\%) } &,- 238 & ,065 &,- 149 & - &, 000 \\
\hline & & & & 3,64 & \\
\hline & & & & 4 & \\
\hline
\end{tabular}

Fonte: Elaborada pelos autores com base nos resultados da pesquisa

Desta forma, o modelo demonstra um indício de que as compras públicas podem ser utilizadas como instrumento de desenvolvimento local nos municípios do Paraná. Além do benefício do produto/serviço adquirido pela administração pública, as compras públicas incentivam o mercado local, fomentando o desenvolvimento.

Segundo o modelo estimado, o aumento de $R \$ 1.000,00$ nos gastos em compras públicas em fornecedores locais por habitante implica no aumento de 0,006 no IPDM, considerando a escala de 0 a 1 . Salienta-se que apesar do modelo demonstrar uma relação positiva entre a realização de compras públicas em fornecedores locais e o IPDM, verifica-se que o impacto das compras públicas no IPDM é de pequena proporção. Desta forma, a utilização das compras públicas como fator estratégico para desenvolvimento socioeconômico aparenta suas limitações.

Os resultados convergem aos estudos apresentados na revisão de literatura, demonstrando mais uma possível aplicação das compras públicas como fator estratégico. Contudo, ressalta-se a necessidade de aprofundar estudos acerca do tema, sendo este estudo limitado apenas aos municípios paranaenses.

Destaca-se que o processo de compras públicas está subordinado a Lei ํㅡㄴ 8.666/93, sendo necessários a análise jurídica da possibilidade de aplicação de critérios que favoreçam os fornecedores locais. Outro ponto importante de discussão é qual a efetividade das compras em fornecedores locais no desenvolvimento. Até 
qual ponto os ganhos no desenvolvimento econômico local compensam uma possível menor eficiência financeira municipal. Os resultados apontam uma relação positiva, contudo, necessita-se de mais estudos empíricos e de uma maior discussão quanto ao tema junto a sociedade.

\section{CONSIDERAÇÕES FINAIS}

O modelo estimado demonstra que as compras públicas, quando realizadas em fornecedores locais, implicaram em um maior IPDM nos municípios paranaenses em 2013. Os resultados contribuem para a discussão quanto a utilização das compras públicas como fator estratégico.

As compras públicas vêm sendo foco de diversas políticas públicas como instrumento de fomento. A recente reforma da lei de licitações para inclusão fomento de produtos ambientalmente sustentáveis e 0 fortalecimento de pequenos produtores rurais são exemplos. Contudo, a utilização das compras públicas como indutor do desenvolvimento local necessita de um maior debate e da adequação da legislação vigente referente ao processo de licitação.

Desta forma, indica-se a replicação deste estudo para demais unidades federativas e o estudo de possíveis medidas tomadas para fomento das compras visando maximizar o desenvolvimento local sem comprometer a eficiência econômica da entidade pública prevista na legislação acerca das compras públicas.

\section{REFERÊNCIAS}

BRASIL. Ministério do Planejamento, Orçamento e Gestão (MPOG). 2012.Compras sustentáveis na administração pública ganham em eficiência econômica. Disponível em: $\quad$ http://cpsustentaveis.planejamento.gov.br/noticias/compras-sustentaveis-naadministracao-publica-ganham-em-eficiencia-economica. Acesso em: 21 jul. 2016.

BRASIL. Lei no8.666 de 21 de junho de 1993. Regulamenta o art. 37, inciso XXI, da Constituição Federal, institui normas para licitações e contratos da Administração Pública e dá outras providências. In: Diário Oficial da República Federativa do Brasil, Brasília, DF, 22 jun. 1993.

CALDAS, E. de L.; NONATO, R. S. Compras públicas e promoção do desenvolvimento local. Revista do Serviço Público, n. 64, jan/jun. 2013.

CHAVES, Fernanda Rodrigues Drumond; BERTASSI, André Luís; SILVA, Gustavo Melo. Compras Públicas e Desenvolvimento Local: micro e pequenas empresas locais nas licitações de uma universidade pública mineira. Revista de Empreendedorismo e Gestão de Pequenas Empresas, v. 8, n. 1, p. 77-101, 2019.

EDLER, J.; GEORGHIOU, L.; BLIND, K.; UYARRA, E. Evaluating the demand side: New challenges for evaluation. Research Evaluation, n. 21, fev. 2012.

EUROPEAN COMMISSION. 2005. Public procurement for research and innovation: Developing procurement practices favourable to R\&D and innovation. Disponível em: http://ec.europa.eu/invest-in- 
research/pdf/download_en/edited_report_18112005_on_public_procurement_for_res earch_and_innovation.pdf. Acesso em: 21 jul. 2016.

FÁVERO, L. P.; BELFIORI, P.; SILVA, F. L. da; CHAN, B. L. Análise de dados: modelagem multivariada para tomada de decisões. Rio de Janeiro: Elsevier, 2009.

INSTITUTO BRASILEIRO DE GEOGRAFIA E ESTATÍSTICA (IBGE). 2016. Sistema IBGE de Recuperação Automática (SIDRA). Disponível em: http://www.sidra.ibge.gov.br/640 . Acesso em: 05 jul. 2016

INSTITUTO PARANAENSE DE DESENVOLVIMENTO ECONÔMICO E SOCIAL (IPARDES). 2016. Base de Dados do Estado. Disponível em: http://www.ipardes.pr.gov.br/imp/index.php . Acesso em: 04 jul. 2016.

LOPES, M. N.; OLIVEIRA, V. C. F. de. Compras Públicas sustentáveis. In: CONSAD de Gestão Pública, 5. Brasília - DF. Anais... Brasília, 2012.

SERVIÇO BRASILEIRO DE APOIO ÀS MICRO E PEQUENAS EMPRESAS (SEBRAE). 2016. Compra PR. Disponível em: http://www.comprapr.com.br/ . Acesso em: 04 jul. 2016. 